\title{
Avaliando as competências escolares através da Prova Brasil usando ferramenta web ${ }^{1}$
}

\author{
Rodrigo Guedes Pereira Pinheiro ${ }^{1}$, Marcos Elia ${ }^{2}$, Fábio F. Sampaio ${ }^{3}$ \\ NCE - Instituto Tércio Pacitti de Aplicações e Pesquisas Computacionais - \\ Universidade Federal do Rio de Janeiro (UFRJ) \\ Caixa Postal 15.064 - 21.941-909 - RIO DE JANEIRO - RJ - Brazil \\ rodgpp@gmail.com, melia@nce.ufrj.br, ffs@nce.ufrj.br
}

\begin{abstract}
In the early 90's Brazil joined the group of countries that adopt external evaluations as a systematic means of generating information for policy formulation in different educational levels. This research work aims at raising awareness and preparing teachers to pursue the practice of school evaluation. In this sense, we are putting in the hands of principals and teachers a web tool with statistic data that would allow them to analyze the result of their students' academic performance and the intersection of this result with the analysis of competences, using data results of "Prova Brasil" applied by Inep in 2007, 2009 and 2011.
\end{abstract}

Resumo. No início dos anos de 1990, o Brasil adotou as avaliações externas como um meio sistemático de gerar subsídios para a formulação de politicas nos diferentes níveis educacionais. Esta pesquisa tem como motivação sensibilizar e preparar os professores para o exercício da prática da avaliação escolar. Assim, estamos colocando nas mãos dos diretores e professores uma ferramenta web com dados estatísticos que lhes permita analisar o resultado do desempenho escolar de seus alunos e do cruzamento desse resultado com a analise das competências, tomando como base de dados os resultados da Prova Brasil aplicada pelo Inep em 2007, 2009 e 2011.

\section{Introdução}

No início da década de 1990, o Brasil através do SAEB - Sistema de Avaliação da Educação Básica, entrou para o grupo de países que adota as avaliações externas como um meio sistemático de gerar subsídios para a formulação de políticas nos diferentes níveis educacionais [Vianna 2003].

Até então, a única forma de avaliação externa que se tinha no país com o propósito de avaliar a educação em um determinado nível educacional, no caso o nível médio, era o exame de vestibular, o qual obviamente era inadequado por ser um exame

1 Trabalho parcialmente financiado pelo Programa governamental PROUCA, através do Edital MCT/CNPq/CAPES/MEC-SEB n 76/2010 
de entrada para o $3^{\circ}$ grau e, portanto, com características diferentes de um exame diagnóstico de saída do $2^{\circ}$ grau. Infelizmente, o Exame Nacional de Ensino Médio (Enem), criado exclusivamente com este fim sob orientação do Inep-Instituto Nacional de Estudos e Pesquisas Anísio Teixeira (http://inep.gov.br), sucumbiu aos interesses políticos pragmáticos, perdendo suas características de um exame diagnóstico e, por ironia, percorrendo um caminho de volta para um único e centralizado vestibular nacional [Vianna 1993].

Por ora, ainda permanece intocado em suas características originais o SAEB, também uma iniciativa do Inep, formado por um conjunto de instrumentos e índices denominado Prova Brasil que permite um diagnóstico da situação dos alunos, professores e das escolas do ensino básico brasileiro.

O presente trabalho de pesquisa tem como motivação sensibilizar e preparar os professores para o exercício da prática da avaliação escolar, seja levando-os a melhorar os instrumentos e procedimentos de avaliação que usa em sala de aula, seja aprimorando sua percepção sistêmica sobre os fatores condicionantes, externos e internos, do sucesso escolar, o que só pode ser alcançado pela análise dos resultados de uma avaliação externa como, por exemplo, aqueles gerados pela Prova Brasil.

Ele faz parte de uma das metas do projeto "Uca na Cuca" [Sampaio e Elia 2012] sobre o uso de Robótica Educacional no âmbito do programa PROUCA (2010) do governo federal.

Este artigo está organizado em quatro seções, incluindo esta seção introdutória. Na seção 2, serão dados detalhes sobre o que denominamos "Avaliação da Avaliação Escolar" que inclui avaliações internas, referentes às práticas avaliativas dos professores em sala de aula, e externas, no caso, feitas a partir da Prova Brasil. Na seção 3, apresentaremos a ferramenta construída por meio de uma primeira versão exploratória em macros em uma planilha Excel, ilustrando suas funcionalidades através de exemplos e depois na construção de uma aplicação em um ambiente Web. E na seção 4, apresentaremos nossas considerações finais seguidas das referências bibliográficas citadas e consultadas.

\section{Avaliação da avaliação escolar}

Uma análise crítico-reflexiva sobre as questões relacionadas ao problema educacional brasileiro, tais como escola e a sociedade, políticas públicas educacionais, desvalorização do fazer docente, desvalorização do espaço escolar, escola e responsabilidade social, para citar as mais importantes; poderia nos levar rapidamente às seguintes constatações:

1. Mudanças educacionais não se fazem apenas com boas intenções e voluntarismo.

2. O problema da educação não pode ser resolvido apenas no âmbito do microcosmo da escola e do esforço individual de cada um.

3. Há necessidade de um esforço coletivo e coordenado por parte dos setores organizados da sociedade, de fora para dentro das escolas, e de um esforço no sentido oposto por parte dos agentes diretamente envolvidos com o processo educacional, em busca de um ponto de equilíbrio, ao qual poderíamos denominar de ecossistema escolar de educação sustentável. 
E para sermos consequentes diante dessas constatações, deveríamos concluir também que o processo de avaliação escolar tem que ser revisto e levado mais a sério em todas as suas instâncias, tanto aquelas feitas internamente pelos professores das escolas, quanto àquelas feitas externamente pelos órgãos governamentais, universidades, ou institutos de pesquisa.

Acreditamos que procedendo assim estaremos contribuindo para o resgate do que a professora Therezinha Rios denomina competência técnica e política do professor [Rios 2001]. No primeiro caso, tem-se a competência técnica para avaliar bem o desempenho escolar de seus alunos, porque uma reflexão sobre o processo de avaliação leva rapidamente a uma reflexão crítica sobre a prática docente em sala de aula. E a competência política, por exemplo, para compreender criticamente o papel da escola e fazer valer seus direitos e valores profissionais junto às autoridades e à sociedade.

\subsection{Avaliações internas: práticas avaliativas em sala de aula}

A literatura já consolidada sobre avaliação escolar mostra-nos que aquela que é feita pelos professores em sala de aula é bastante complexa, por um lado, devido à variabilidade de propósitos e métodos envolvidos e, por outro, porque em sala de aula o professor- além de ensinar bem - deve também avaliar bem.

Ao darmos foco à "Avaliação da Avaliação Escolar", instrumentalizando o professor com ferramentas TIC que contribuem para a melhoria da sua prática avaliativa, estamos nos apoiando nas ideias de Eric Rogers para quem a forma de avaliar de um professor demonstra muito da forma que ele ensina:

"Let me listen to your examiners making their examination question and let me listen again when they are marking the answers, and $i$ shall know the real value and promise of your work". [Eric Rogers, apud Jennison 1994]

Assim, para este excelso físico britânico que se dedicou à Educação para Ciência e que ao ser agraciado pela AAPT (American Association of Physiscs Teachers) com a medalha Oersted (1969) apresentou uma conferência sob o título "Examinations: Powerful Agents for Good or Ill in Teaching" [Rogers 1969], é na maneira de avaliar que aparece tudo o que é importante para o professor. Avaliando a avaliação é uma forma de identificar crenças e valores pedagógicos do professor, bem como a forma pelo qual o conteúdo foi ensinado.

Portanto, promovendo a "Avaliação da Avaliação Escolar" juntamente com os professores, acreditamos não só poder contribuir para sua competência técnica no processo de ensino-aprendizagem-avaliação, mas também para sua competência política (visão crítica) em sua prática, de seus colegas e de outros agentes internos e externos à escola. Neste ponto, estamos concordando com Therezinha Rios [Rios 2001] que defende que estas duas competências são os pilares da ética educacional.

\subsection{Avaliações externas: Prova Brasil}

A Prova Brasil e o Saeb são constituídos de dois instrumentos de avaliação. O primeiro é um questionário socioeconômico, composto de várias perguntas, destinado a professores, gestores e alunos. Até o presente momento foram realizadas três avaliações em 2007, 2009 e 2011. Os questionários dos professores e gestores foram construídos segundo sete grandes categorias: formação do professor (apresenta perguntas que analisam a experiência na educação e qualificação profissional); sobrecarga didática 
(verificam a carga horária de professores e gestores no exercício de suas atividades, além da participação em cursos de formação continuada e em trabalhos fora do magistério); projeto pedagógico (analisam as percepções sobre as técnicas pedagógicas adotadas pela escola entre os seus agentes: alunos, professores e gestores); infraestrutura TIC (utilização de recursos tecnológicos como computador, projetores, bem como ferramentas necessárias para a realização das atividades na escola); violência (avaliam ambiente de insegurança física e o comportamento dos agentes que envolvem a escola); políticas públicas \& gestão (como a escola realiza as políticas públicas estabelecidas pelo governo) e os livros didáticos (a visão apresentada por professores e gestores sobre a adequação do material utilizado pelos alunos).

O questionário dos alunos foi construído segundo quatro dimensões com um total de 44 perguntas, a saber: o perfil do aluno (qual sua idade, qual seu sexo, entre outros); informação econômica ("na sua casa tem televisão em cores", "possui vídeo cassete ou DVD", entre outros); perfil da família ("Você mora com sua Mãe?", "Até que série sua mãe estudou?", entre outros); histórico escolar ("Você já foi reprovado antes?", "O professor corrige o dever de casa de língua portuguesa?").

O segundo instrumento de avaliação é constituído por provas aplicadas aos alunos referentes às disciplinas de Português e Matemática das últimas séries de cada seguimento ( $5^{\circ}$ e $9^{\circ}$ Anos). A Prova Brasil tem como unidade de observação o número total de alunos por turma. Só participaram da pesquisa turmas com mais de 20 alunos nas disciplinas avaliadas.

Na última versão da prova Brasil, 2011, novos dados detalhados das provas de Portugês e matemática foram disponibilizados, exibindo os erros e acertos dos alunos por item de prova e as suas relações com as competências apresentadas nas questões.

\section{Apresentação da Ferramenta}

A ferramenta que está sendo proposta servirá como um importante instrumento de auxílio aos professores e gestores no dia-a-dia escolar. Ela utiliza dados importados da base de dados do Inep, referentes ao questionário sócioeconômico aplicado aos diretores, professores e alunos, bem como aos dados da prova Brasil: disciplinas de Português e Matemática das últimas séries de cada seguimento ( $5^{\circ}$ e $9^{\circ}$ Anos); correspondentes aos anos de 2007, 2009 e 2011.

As funcionalidades da ferramenta englobam (Figura 1), além de estatísticas clássicas como a descritiva e a análise de itens, outras com caráter inovador: (i) Análise das Competências e Habilidades Associadas (ACHA) descritas na matriz de referência da Prova Brasil e (ii) Ecossistema Escolar (Eco@E), uma análise que relaciona o desempenho dos alunos (Português e Matemática) com os dados dos questionários socioeconômicos de seus professores e de diretores segundo as sete grandes categorias descritas na seção 2.2.

As análises ACHA e ECO@E e o desenvolvimento da ferramenta Web fazem parte de uma pesquisa de dissertação com o propósito de apoiar a prática da avaliação escolar por professores. Contudo, devido ao espaço limitado, apenas será dado foco neste artigo à análise ACHA. 


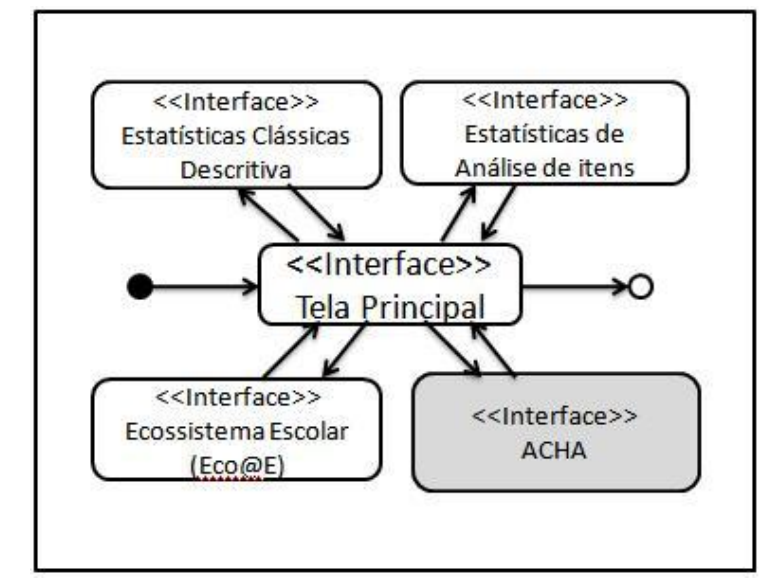

Figura 1. - Diagrama da Interface da ferramenta

\subsection{Levantamento de requisitos}

A especificação da ferramenta em tela foi feita com base na experiência adquirida com o intuito de desenvolver o modelo da análise Ecossistema Escolar (Eco@E). Essas pesquisas foram feitas utilizando diferentes ferramentas (SPSS, Access, Excel, etc.) de forma assistemática, e uma vez encontrado um modelo satisfatório, decidiu-se automatizá-lo e torná-lo disponível aos profissionais de educação.

Um aspecto importante desta decisão que deve ser registrado no presente trabalho é o caminho percorrido através da utilização de planilhas eletrônicas e macros disponíveis em aplicativos comerciais para as análises, antes de partir para a escolha do uso de aplicações na Web com banco de dados e do uso de soluções mais sólidas envolvendo plataformas computacionais e pacotes estatísticos, tais como $\mathrm{SPSS}^{2}$ e $\mathrm{R}^{3}$.

Um dos principais desafios da ferramenta, em planilhas eletrônicas, é a organização dos dados estudados. O volume é grande, pois consiste em dados gerados por todas as escolas da rede pública de todo o Brasil nos anos 2007, 2009 e 2011, e tal fato pode gerar um problema de desempenho da ferramenta. Para resolver a questão, inicialmente, foi necessário pensar em uma solução onde os dados ficassem separados e indexados em vários arquivos, com o propósito de acelerar a busca da informação e a não oneração da memória do computador.

Chegou-se a pensar em disponibilizar as bases de dados em servidor web com tecnologia de Web Service e uma interface local através de macros no MS Excel para conectar esta base de dados. Esta solução, no entanto, implicaria na necessidade dos usuários possuir, no momento da utilização da ferramenta, de acesso à internet de banda larga à arquivo muito grande de dados, o que, não corresponde ainda à realidade da maioria das escolas do Brasil.

Apos esta fase inicial de estudo dos dados, foi percebido que as informações nas planilhas eletrônicas apresentavam uma performance não satisfatória, necessitando de uma outro solução. Para resolver esse problema foi desenvolvida uma nova ferramenta, agora, na Web onde os dados foram armazenados em um banco de dados. O escolhido foi o MySQL [McLaughlin 2013], com grande capacidade de processamento de dados, acessados em uma aplicação desenvolvida no software Ruby on Rails [Urubatan 2009],

\footnotetext{
${ }^{2}$ SPSS é um software estatístico para as ciências sociais.

${ }^{3} \mathrm{R}$ é uma linguagem e ambiente para computação estatística e gráficos.
} 
ferramenta para o desenvolvimento rápido de sistemas para Web. Dentre os resultados positivos desta escolha podemos destacar: a utilização de software livre não agregando custos adicionais ao projeto; o uso de um $\mathrm{SGBD}^{4}$ implicando numa diminuição no tempo de recuperação de dados e geração de resultados; facilidade nas atualizações da ferramenta, uma vez que a aplicação está na Web; usuários não necessitarão de muitos recursos de máquina somente um Browser e um acesso a internet.

Outro fato importante ocorreu no processo de tratamento dos dados, onde primeiramente foi feito a importação das últimas três pesquisas da Prova Brasil no site do Inep, download dos microdados, em seguida realizado sucessivos tratamento nos dados gerando um novo modelo de informação. Foi criado um conjunto de tabelas com valores previamente calculados, para isso foi utilizado os softwares estatístico SPSS e o de mineração de dados $\mathrm{Weka}^{5}$ em conjunto com SGBD MySQL. O objetivo deste procedimento foi evitar que a cada solicitação feita pelo usuário por informações iniciase uma série de rotinas de cálculos onerando o processamento das informações prejudicando o desempenho da ferramenta.

Atualmente encontramos algumas ferramentas disponíveis na Internet com o propósito de apresentar os dados da Prova Brasil de maneira mais fácil, podemos citar como exemplo o site QEdu, [Oliveira et al 2013], mas sem apresentar um novo modelo para as informações do Inep.

\subsection{Interface e serviços da ferramenta ACHA}

A ferramenta ACHA que será apresentada nesta seção permite o estudo das competências e habilidades adquiridas pelos alunos, suas associações com os resultados alcançados da escola e, bem como, sua classificação em uma determinada escala de avaliação definidas pelo Inep. O sistema é composto por uma tela inicial contendo cinco abas onde é possível realizar pesquisas com um grande número de informações. $\mathrm{O}$ diagrama da (figura 2) apresenta a estrutura da interface.

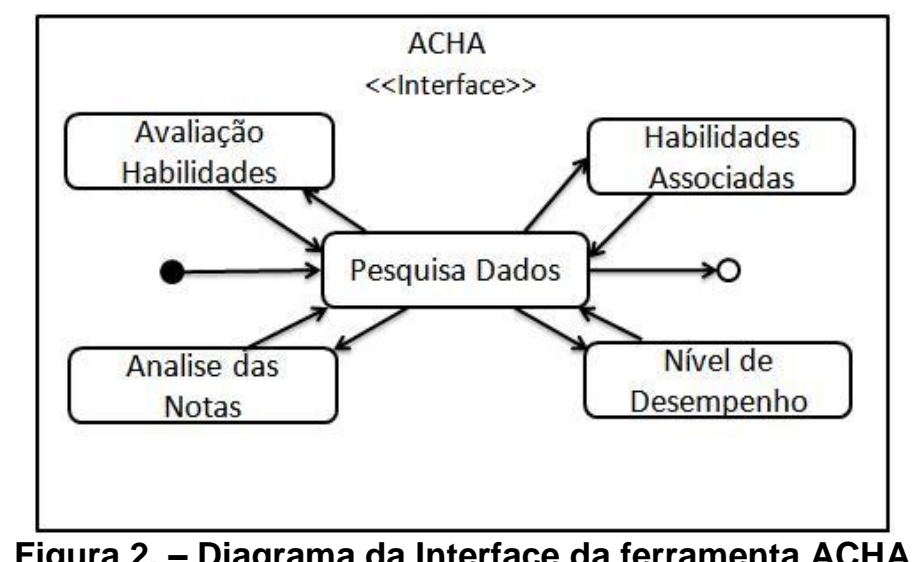

A Interface da pesquisa de dados (Figura 3), é a responsável pela busca de dados disponibilizados pelo Inep. Ela está organizada em um conjunto de três caixas de

\footnotetext{
4 Sistema de Gerenciamento de Banco de Dados - É o conjunto de programas de computador (softwares) responsáveis pelo gerenciamento de uma base de dados.

5 O pacote de software Weka (Waikato Environment for Knowledge Analysis) procede à análise computacional e estatística dos dados fornecidos recorrendo a técnicas de mineração de dados tentando, indutivamente, a partir dos padrões encontrados gerar hipóteses para soluções.
} 
combinação relacionadas que, de forma dinâmica, apresentam várias combinações de consultas. A cada escolha do usuário, novas opções compatíveis com a escolha anterior são exibidas alem de três caixas de seleção para delimitação do contexto da pesquisa. No exemplo apresentado o usuário escolheu os dados de uma escola hipotética com o nome Escola Municipal ABC localizada na cidade de Niterói no Estado do Rio de Janeiro selecionou ainda informações dos alunos do $5^{\circ}$ ano da disciplina de Matemática. Após a seleção dos dados é necessário clicar no botão enviar para atualizar os dados nas outras abas. Será detalhada cada uma das abas a seguir.

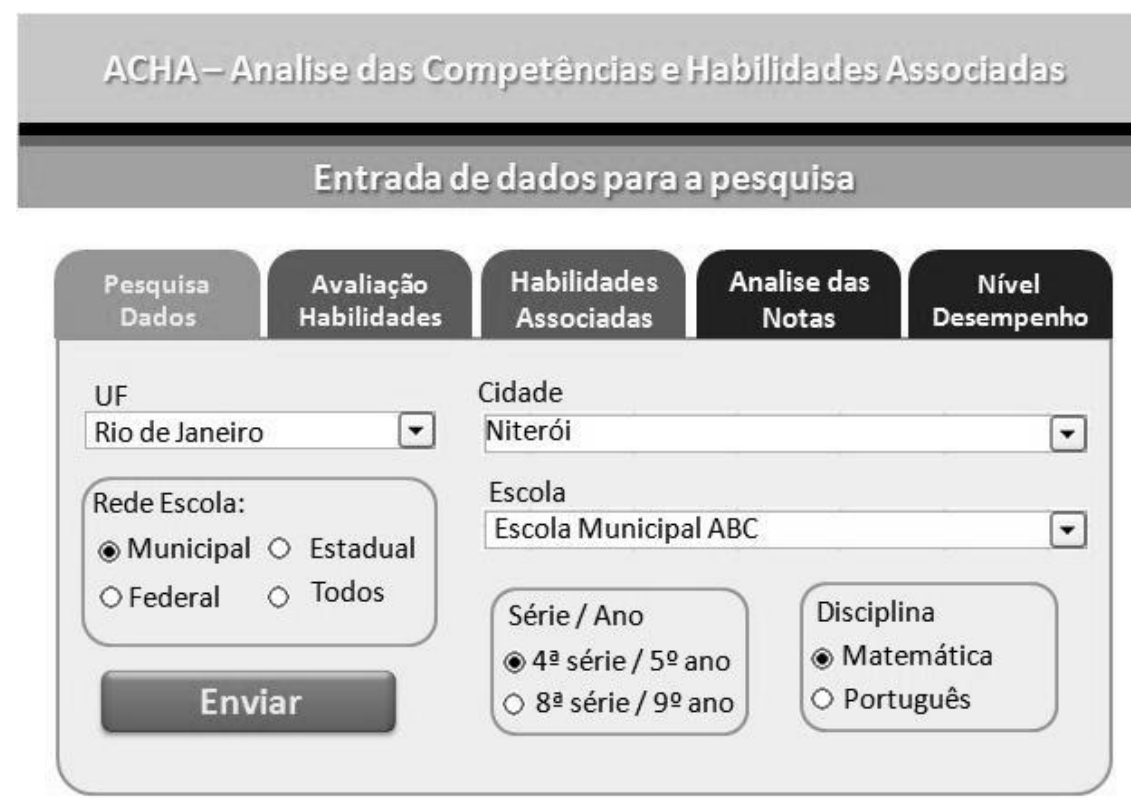

Figura 3. - Tela Pesquisa dados

A aba Avaliação Habilidades (Figura 4) exibirá a lista das habilidades avaliadas na prova Brasil ordenado pelos acertos ou erros cometidos pelos alunos. O calculado é o resultado da divisão entre as questões respondidas, agrupadas por uma determinada habilidade, de forma correta e seu total gerando um índice. No exemplo são apresentados os descritores ordenados pelos melhores índices nas habilidades encontradas. A escola hipotética, Escola Municipal ABC localizada na cidade de Niterói no Estado do Rio de Janeiro, na disciplina de Matemática do $9^{\circ}$ ano, apresentou uma determina lista de temas e descritores ordenados pelo índice de acertos na habilidades testadas.

A aba Habilidades Associadas (Figura 5) exibirá descritores mais relacionados entre si dentro de um determinado contexto. Com estes dados busca-se averiguar se existe relação entre as competências apresentadas com os erros ou acertos cometidos pelos alunos através do resultado gerados pela Prova Brasil. Exemplo, verificar se o aluno que na disciplina de Matemática acerta uma questão sobre frações também acerta uma questão sobre regra de três. Para essa tarefa, como cálculos, foi utilizado a mineração de dados através da técnica da regra de associação. Essa técnica é usada para descobrir elementos que ocorrem em comum dentro de um determinado conjunto de dados. O calculo é complexo e por isso foi utilizado o software de mineração de dados Weka incorporado na aplicação do site desenvolvido através do Ruby on Rails [Urubatan 2009]. Esse procedimento auxiliou nos cálculos desta aba retornando as listas de associações e seu grau de associação. 


\section{ACHA - Analise das Competênojas e Habilidades Assogiadas}

\section{Lista de habilidades ordenadas por descritores certos na prova Inep}

\begin{tabular}{|c|c|c|c|c|c|c|}
\hline \multicolumn{2}{|c|}{$\begin{array}{l}\text { Pesquisa } \\
\text { Dados }\end{array}$} & $\begin{array}{l}\text { Avaliação } \\
\text { Habilidades }\end{array}$ & $\begin{array}{l}\text { Habilidades } \\
\text { Associadas }\end{array}$ & $\begin{array}{r}\text { Analise } \\
\text { Notas }\end{array}$ & $\begin{array}{l}\text { das } \\
s\end{array}$ & $\begin{array}{l}\text { Nivel } \\
\text { esempenho }\end{array}$ \\
\hline \multicolumn{5}{|c|}{$\begin{array}{l}\text { Estado: Rio de Janeiro Cidade:Niterói } \\
\text { Escola:Escola Municipal ABC } \\
\text { Disciplina : Matemática } 4 \text { série/5 ano }\end{array}$} & \multicolumn{2}{|c|}{$\begin{array}{l}\text { Ordem: } \\
\text { Acertos } \\
\text { Erros }\end{array}$} \\
\hline$(\%)$ & Tópico & \multicolumn{3}{|l|}{ Descritor } & Acertos & Total \\
\hline 40 & $\begin{array}{l}\text { Espaçoe } \\
\text { Forma }\end{array}$ & \multicolumn{3}{|c|}{$\begin{array}{l}\text { D1-Identificar a localização/movimentação de objeto, } \\
\text { em mapas, croquis e outras representaçâes gráficas. }\end{array}$} & 40 & 100 \\
\hline 19 & $\begin{array}{l}\text { Espaçoe } \\
\text { Forma }\end{array}$ & \multicolumn{3}{|c|}{$\begin{array}{l}\text { D2-Identificar propriedades comuns e diferenças } \\
\text { entre figuras bidimensionais e tridimensionais, } \\
\text { relacionando-as com suas planificaçỗes. }\end{array}$} & 15 & 80 \\
\hline 10 & $\begin{array}{l}\text { Espaçoe } \\
\text { Forma }\end{array}$ & \multicolumn{3}{|c|}{$\begin{array}{l}\text { D3 - Identificar propriedades de triângulos pela } \\
\text { comparação de medidas de lados e ângulos. }\end{array}$} & 10 & 100 \\
\hline 8 & $\begin{array}{l}\text { Espaçoe } \\
\text { Forma }\end{array}$ & \multicolumn{3}{|c|}{$\begin{array}{l}\text { D4-Identificar relação entre quadriláteros, por meio } \\
\text { de suas propriedades. }\end{array}$} & 4 & 50 \\
\hline 5 & $\begin{array}{l}\text { Espaçoe } \\
\text { Forma }\end{array}$ & \multicolumn{3}{|c|}{$\begin{array}{l}\text { D5 - Reconhecer a conservação ou modificação de } \\
\text { medidas dos lados, do perímetro, da área em ampliaçãa } \\
\text { e/ou redução de figuras poligonais usando malhas } \\
\text { quadriculadas. }\end{array}$} & 4 & $80 \vee$ \\
\hline
\end{tabular}

Figura 4. Aba da Avaliação das Habilidades

A Figura 5 apresenta o resultado da pesquisa da escola fictícia Escola Municipal ABC da cidade de Niterói no Estado do Rio de Janeiro, na disciplina de Matemática do $5^{\circ}$ ano, exibindo os descritores das habilidades e o índice de associação, valor calculado pela regra de associação gerado pelo Weka.

Cabe destacar que para as abas já apresentadas só foram utilizados os dados da Prova Brasil 2011, pois foi somente nesta edição que foram disponibilizados as respostas de cada um dos itens respondidos pelos alunos e as respectivas habilidades possibilitando os cálculos apresentados. Nas abas seguinte foram utilizados todos os estudos realizados pelo Inep (2007, 2009 e 2011)

$\mathrm{Na}$ aba Analise das Notas são exibidos as quatro notas da escola nas disciplinas e séries pesquisadas nos três últimas avaliações realizadas pelo Inep (2007,2009 e 2011) bem como os dados correspondentes as notas municipais, estaduais e nacionais, alem de apresentar em que nível da escala do desempenho a escola se encontra. A escala de desempenho foi desenvolvida pelo Inep. O objetivo desta aba é mostrar aos usuários como a escola se posiciona dentro de um contexto de avaliação externa.

A aba Nível de desempenho busca mostrar em que posição a escola se localiza dentro de três níveis (municipal, estadual e nacional) e nos quatro grupos (combinação entre as duas séries e disciplinas estudadas) nas pesquisas realizadas pelo Inep (2007, 2009, 2011). Essa aba busca apresentar ao usuário um cenário de onde a escola está posicionada quando comparada com outras. 


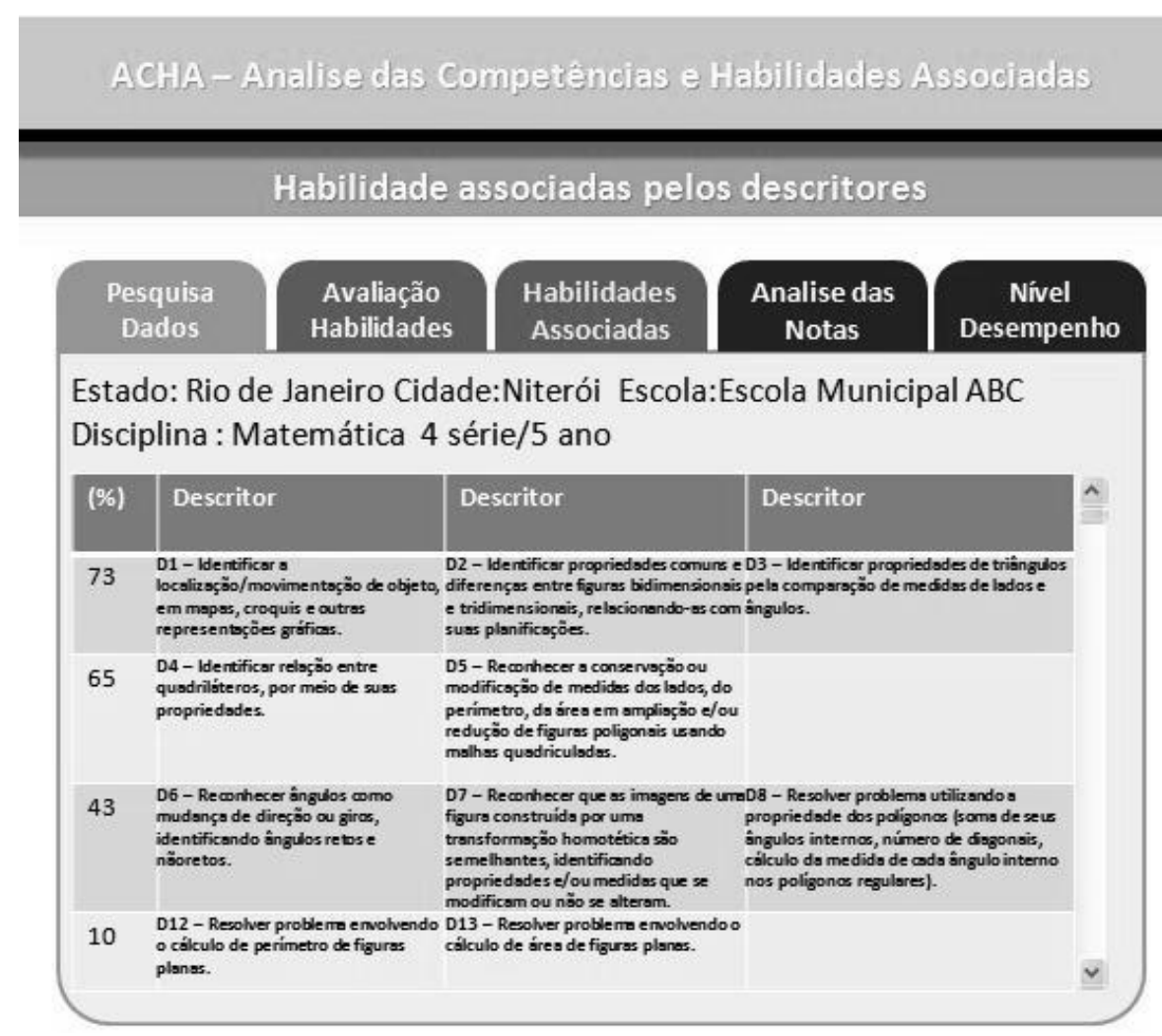

Figura 5. Aba das Habilidades Associadas.

\section{Considerações Finais}

Com este trabalho estamos colocando nas mãos dos diretores e professores uma poderosa - porém operacionalmente simples - ferramenta que lhes permita entender o resultado do desempenho escolar de seus alunos bem como analisar os cruzamento desse resultado, habilidades adquiridas pelos alunos, com os quais, em tese, pode estar relacionados, tomando como base a aplicação da Prova Brasil realizada pelo Inep.

Acreditamos também que a vivência dos professores e diretores com uma análise quantitativa de dados, que dispensa conhecimentos especializados de modelos estatísticos e de linguagem de programação, possa levá-los primeiramente a reconhecerem o valor dos números quando colocados com significado e em contexto adequados e que, pouco a pouco, se apropriem dos conceitos básicos de uma estatística educacional e obtenção de boas medidas educacionais que possam servir de subsídios confiáveis para uma reflexão de suas próprias práticas avaliativas feitas em sala de aula.

\section{Referência}

Brenda Jennison (1994). Wonder and Delight, (Institute of Physics), is a book dedicated to the works and philosophy of Eric Rogers. The book's subtitle is Essays in Science Education in honour of the life and work of Eric Rogers (1902-1990). A biography of Eric Rogers was included.[3]

McLaughlin, Michael (2013). "MySQL Workbench: Data Modeling \& Development Visually Model, Generate, and Manage Databases" Ed. Oralce Press.

Oliveira, Alexandre et al. (2013). “O QEdu”. Disponível em: http://www.qedu.org.br/ 
PROUCA, Edital MCT/CNPq/CAPES/MEC-SEB n $n^{\circ}$ 76/2010

Rios, Terezinha Azerêdo (2001). Ética e competência. 10. Ed. São Paulo: Cortez, 2001. (Coleção Questões da Nossa Época; v.16)

Rogers, Eric M. (1969). "Examinations: Powerful Agents for Good or Ill in Teaching". Am. J. Phys. 37 (10): 954-962. Bibcode: 1969 AmJPh..37..954R. doi: 10.1119 / 1.1975217.

Sampaio e Elia (2012). "Projeto um computador por aluno: pesquisas e perspectivas". Disponível em: < http://www.nce.ufrj.br/ginape/livro-prouca $>$.

Urubatan, Rodrigo (2009).” Desenvolvimento Fácil e Rápido de Aplicações Web Ruby on Rails" .Editora Novatec

Vianna, Heraldo M.(1995). "Avaliação Educacional: uma perspectiva histórica". Revista Estudos em Avaliação Educacional n.12 p. 07-24. Editora Fundação Carlos Chagas

Vianna, Heraldo M.(2003). “Avaliações Nacionais em Larga Escala: análises e propostas". Revista Estudos em Avaliação Educacional n.27 p. 41-76. Editora Fundação Carlos Chagas 